\title{
Synthesis of Imidazolin-2-iminato Titanium Complexes Containing Aryloxo Ligands and Their Catalytic Performance in the Polymerization of $\alpha$ - Olefins
}

\author{
Dana Shoken, ${ }^{\mathrm{a}}$ Linda J. W. Shimon, ${ }^{\mathrm{b}}$ Matthias Tamm, ${ }^{{ }^{*}}$ and Moris S. Eisen ${ }^{\mathrm{a}^{*}}$

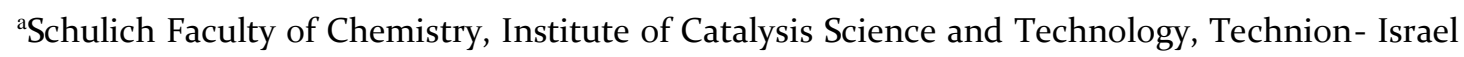 \\ Institute of Technology, Technion City, 32000 Haifa, Israel \\ ${ }^{b}$ Department of Chemical Research Support, The Weizmann Institute of Science, Rehovot 7610o, \\ Israel \\ 'Institut für Anorganische und Analytische Chemie, Technische Universität Braunschweig, \\ Hagenring 30, 38106 Braunschweig, Germany
}

Table of Contents

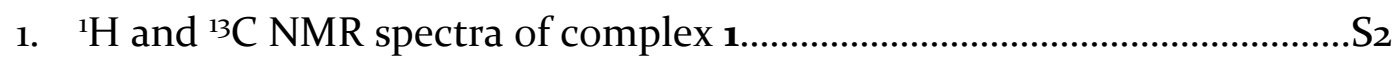

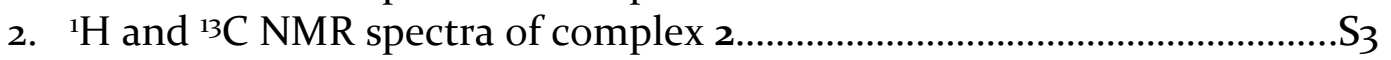

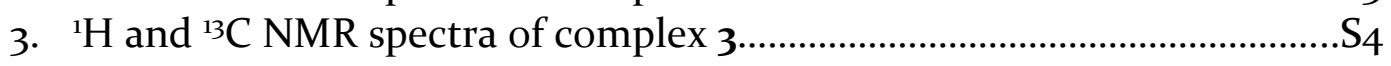

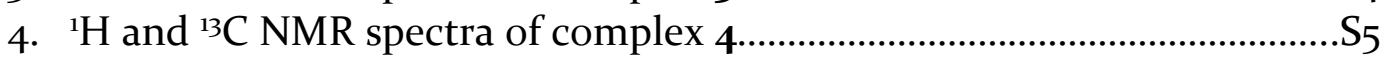

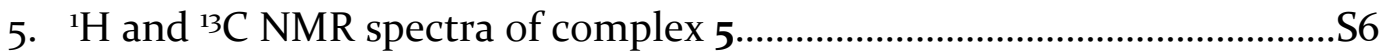

6. Data in the polymerization of propylene, 1-hexene and 1-octene catalysts 1-4 with different co-catalysts....................................................................

7. ${ }^{13} \mathrm{C}$ NMR of atactic polypropylene obtained with complex 1, entry 1 (Table

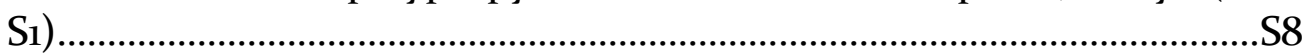

8. ${ }^{13} \mathrm{C}$ NMR of atactic polypropylene obtained with complex 1, entry 3 (Table

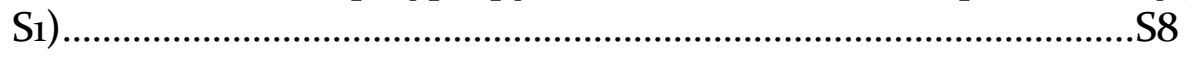

9. ${ }^{13} \mathrm{C}$ NMR of atactic polypropylene obtained with complex 2 , entry 4 (Table $\mathrm{S} 1$ ).

10. ${ }^{13} \mathrm{C}$ NMR of atactic poly(1-hexene) obtained with complex 2 , entry 11 (Table

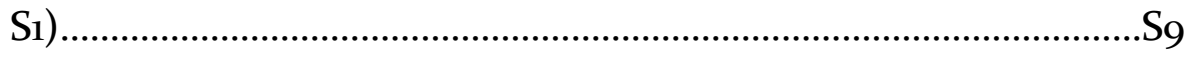

11. ${ }^{13} \mathrm{C}$ NMR of atactic poly(1-octene) obtained with complex 2, entry 17 (Table $\mathrm{S} 1)$....... S10

12. Equations of rate of insertion $\left(\mathrm{R}_{\mathrm{i}}\right)$ and rate of termination $\left(\mathrm{R}_{\mathrm{t}}\right) \ldots \ldots \ldots \ldots . .$. ....... 


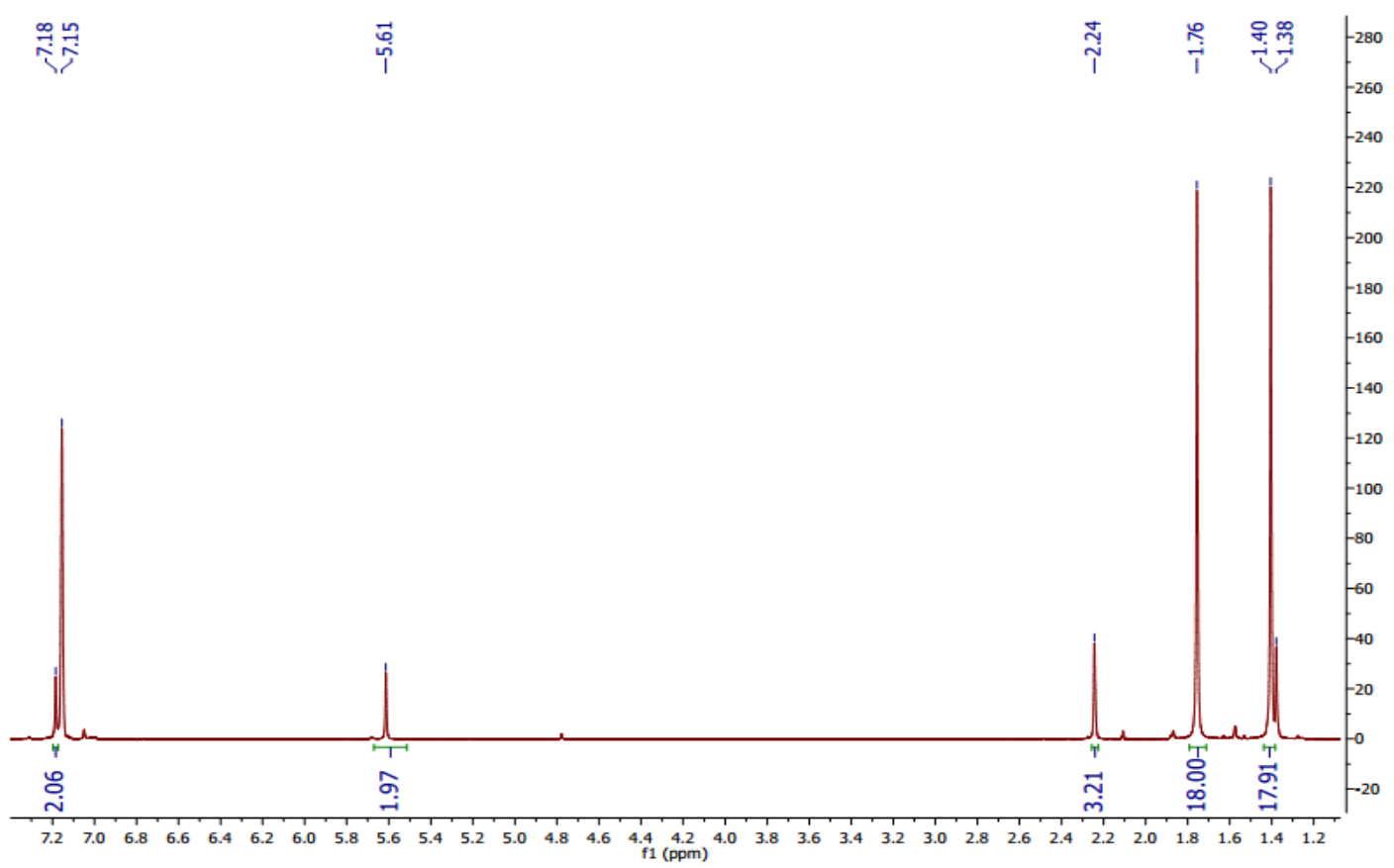

Figure S1. 'H NMR spectra of complex 1.

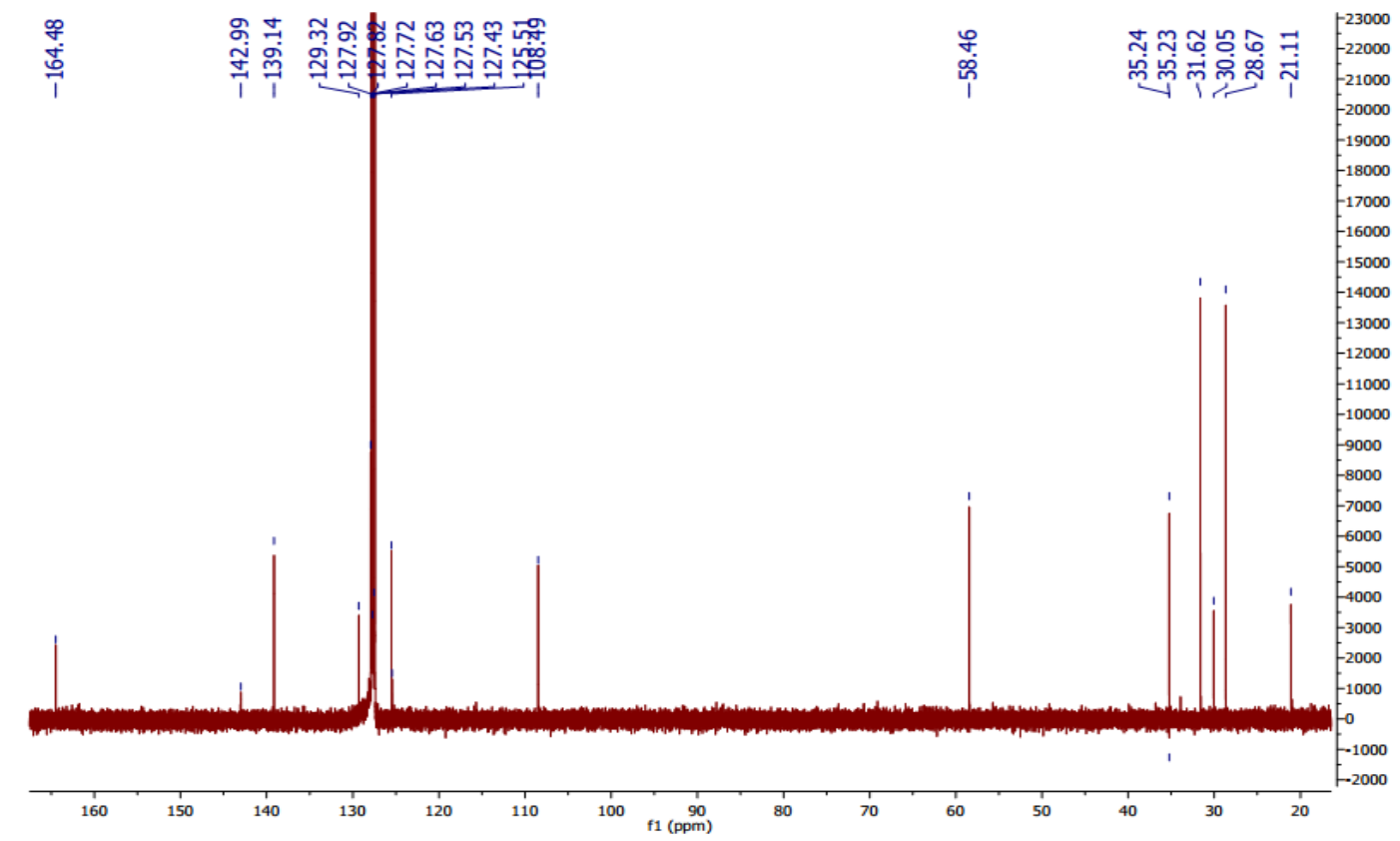

Figure S2. ${ }^{13} \mathrm{C}$ NMR spectra of complex 1. 


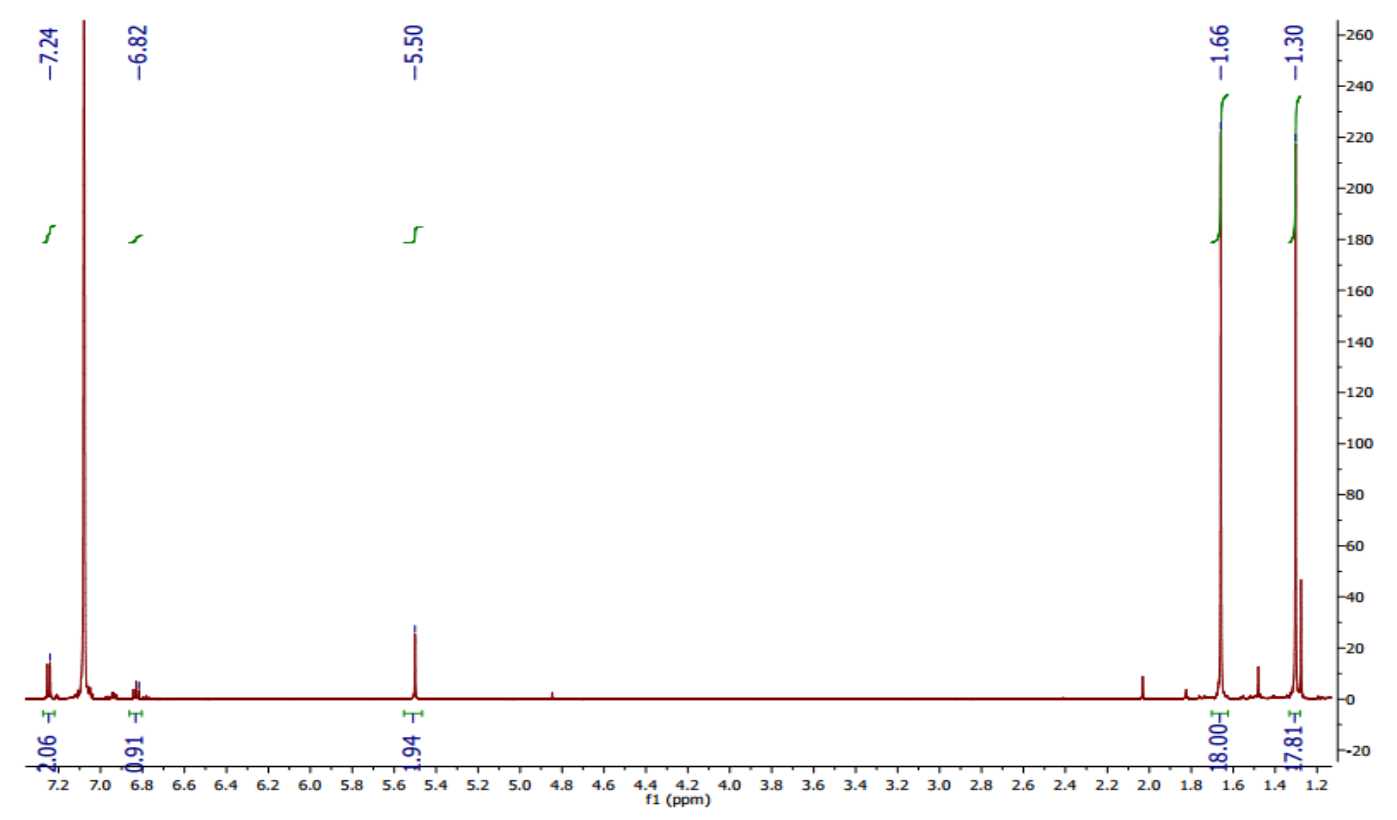

Figure S3. ${ }^{1} \mathrm{H}$ NMR spectra of complex 2.

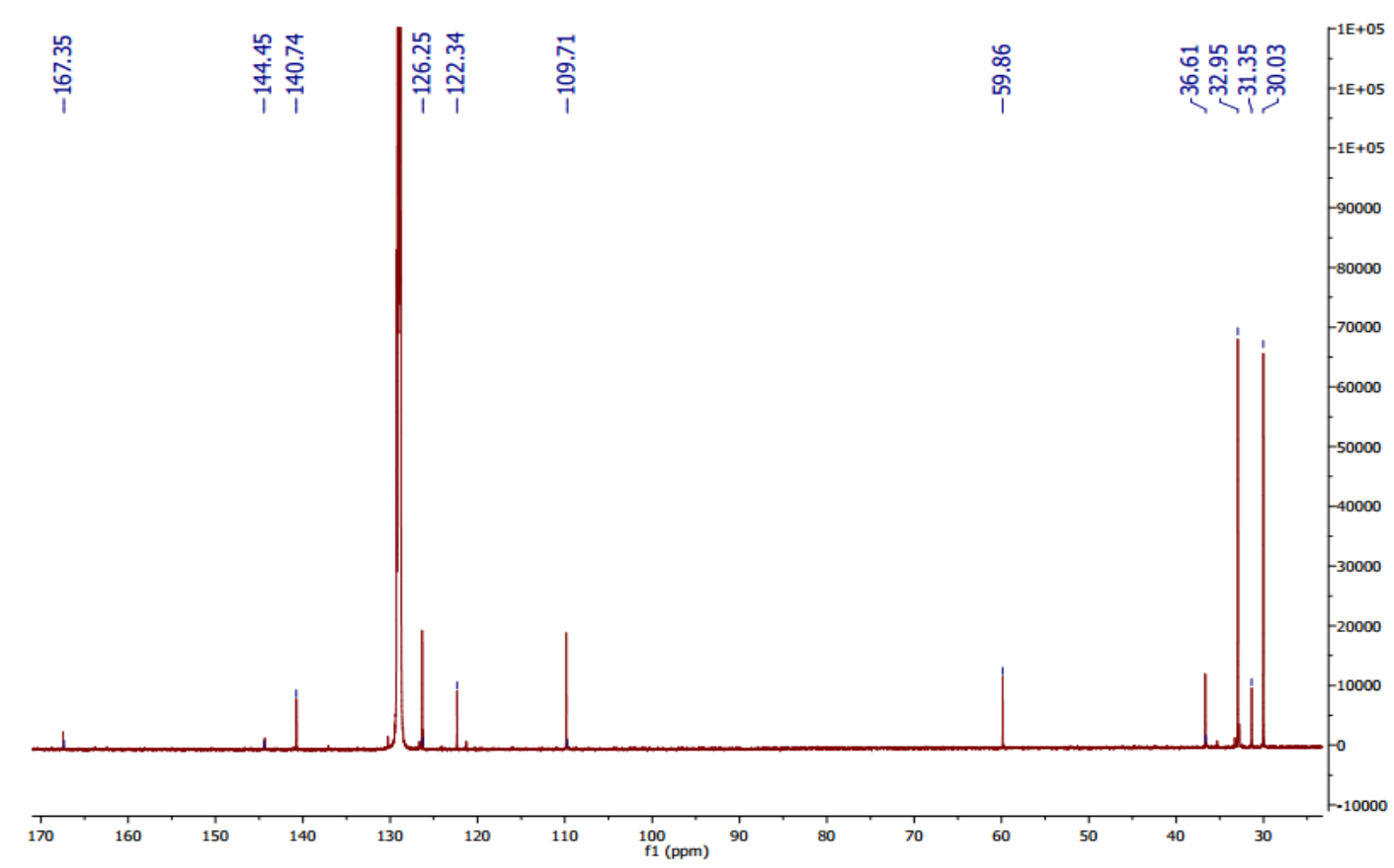

Figure S4. ${ }^{13}$ C NMR spectra of complex 2. 


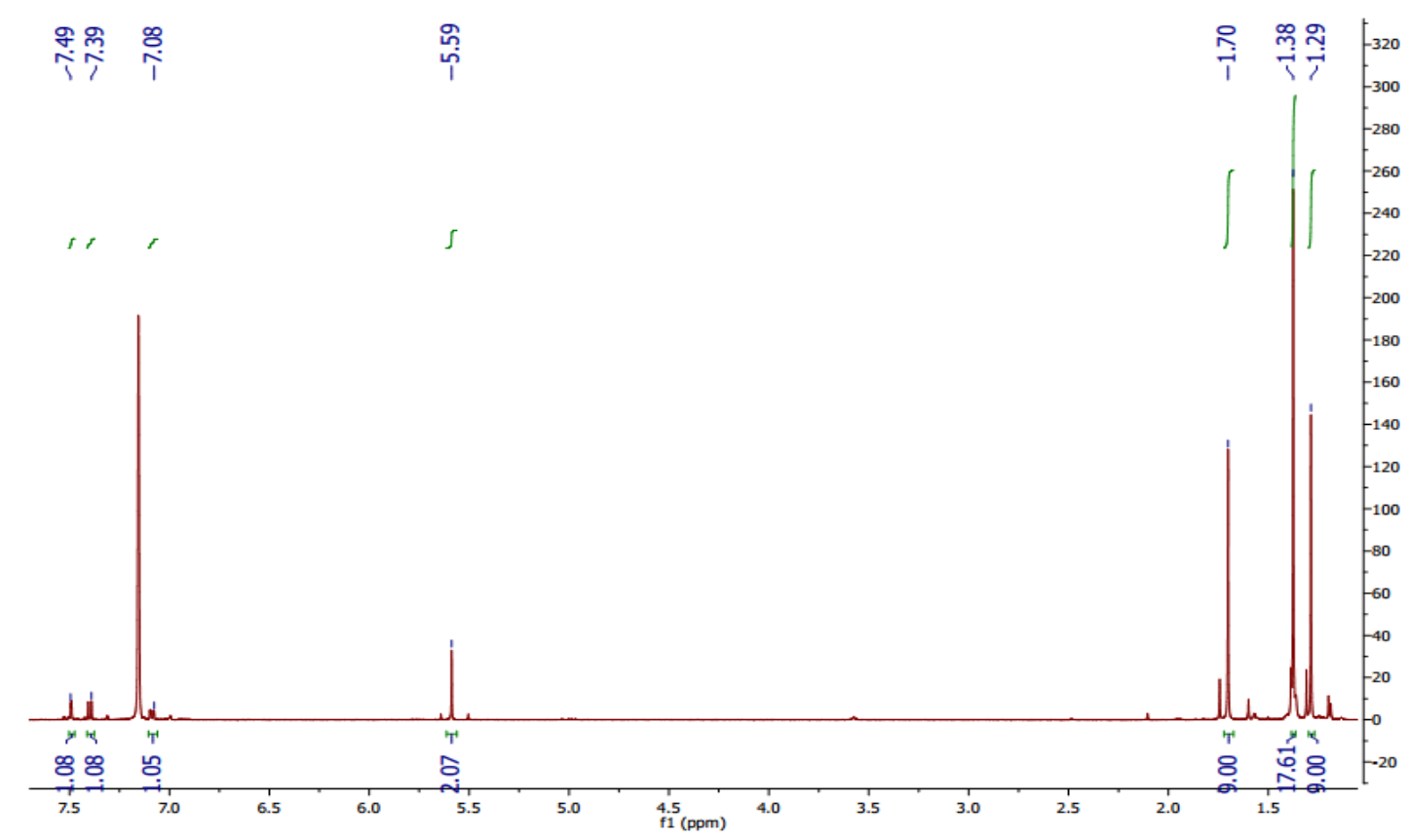

Figure S5. ${ }^{1} \mathrm{H}$ NMR spectra of complex 3.

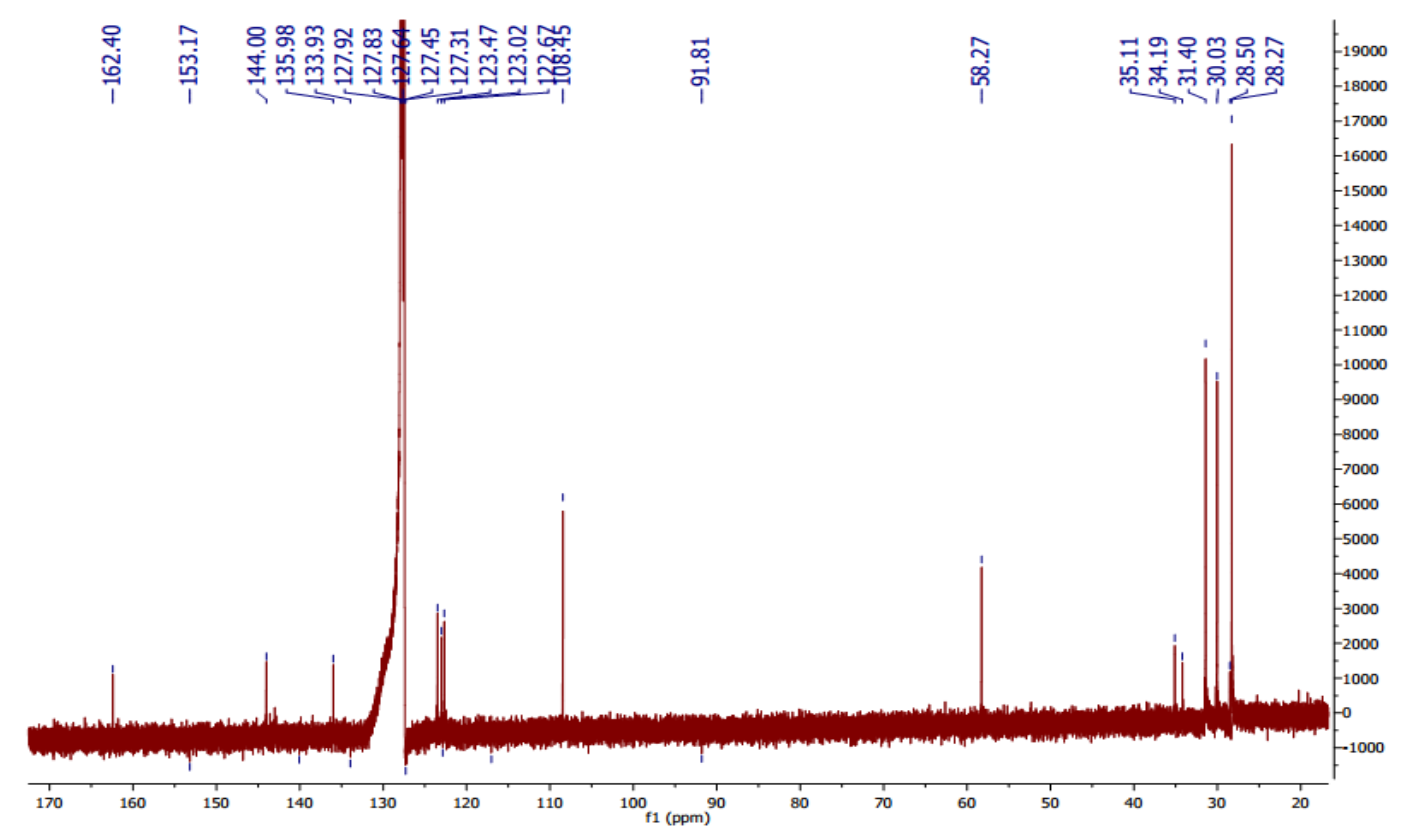

Figure S6. ${ }^{13} \mathrm{C}$ NMR spectra of complex 3. 


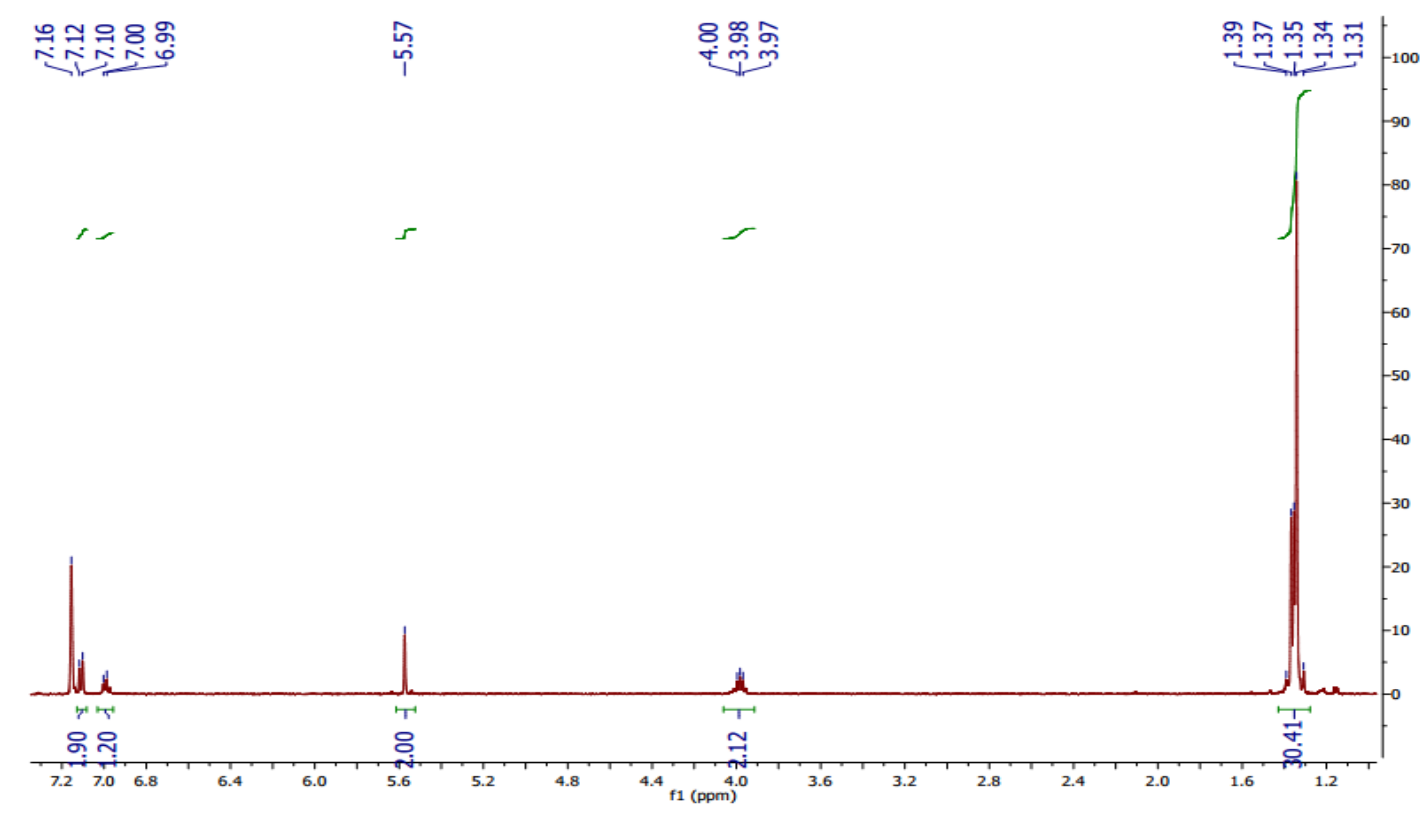

Figure S7. ${ }^{1} \mathrm{H}$ NMR spectra of complex 4.

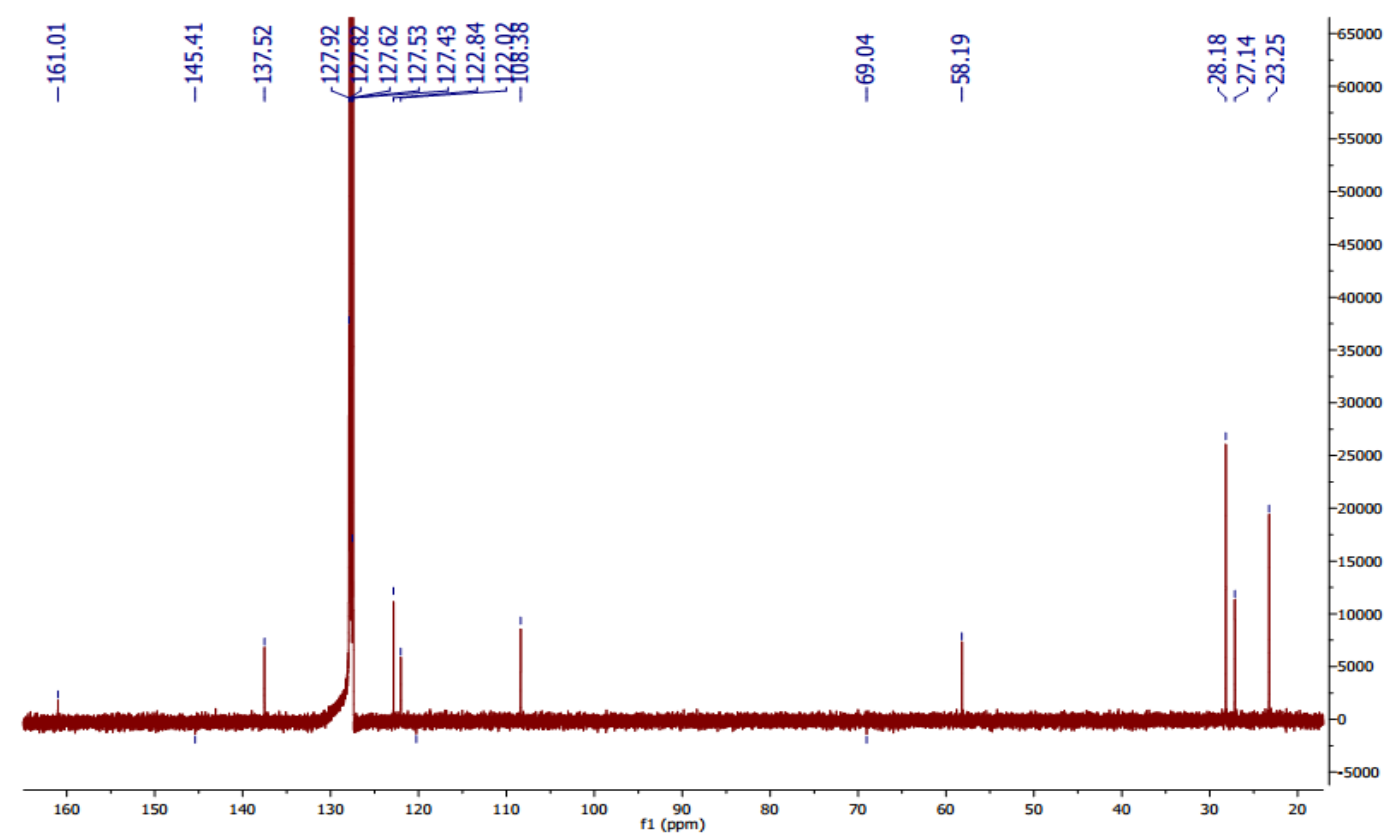

Figure S8. ${ }^{13} \mathrm{C}$ NMR spectra of complex 4. 


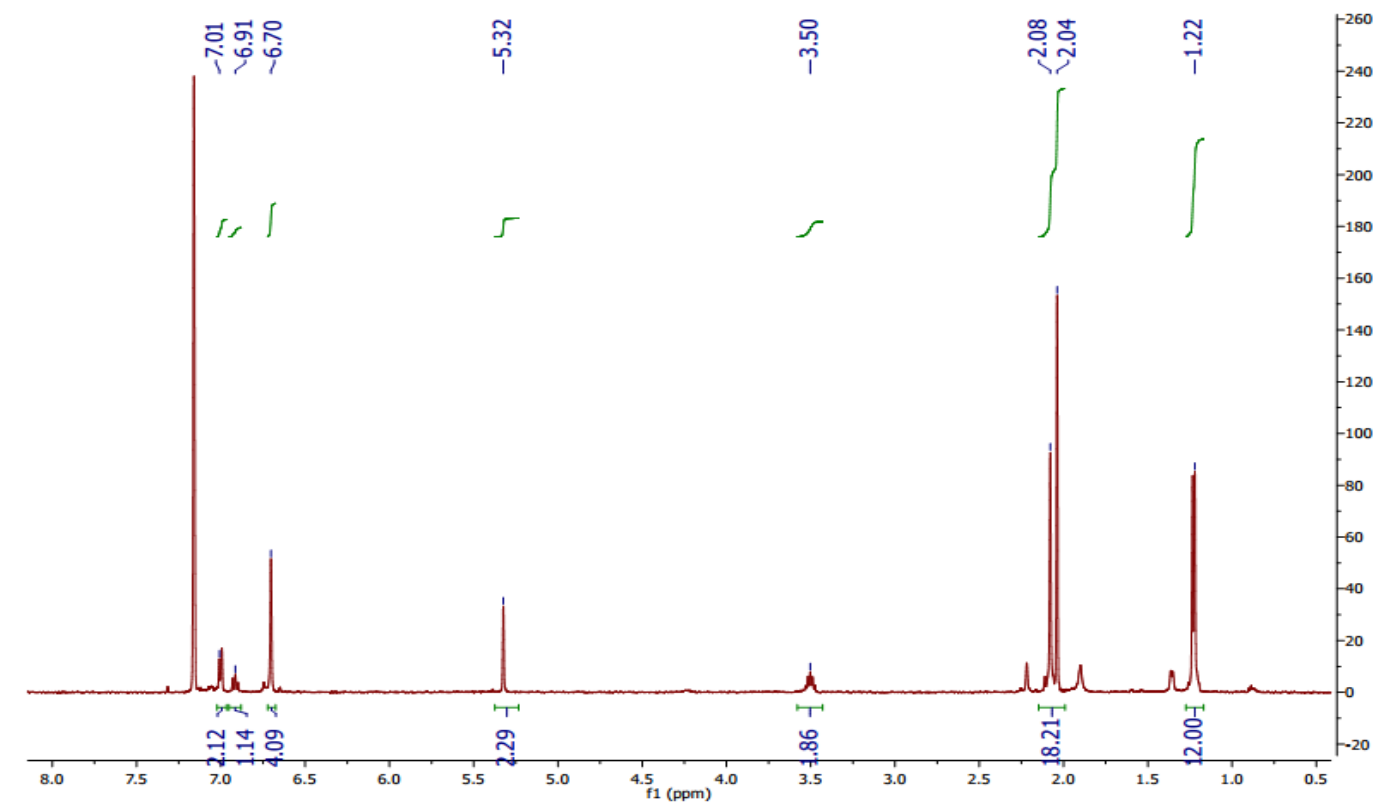

Figure S7. ${ }^{1} \mathrm{H}$ NMR spectra of complex 5.

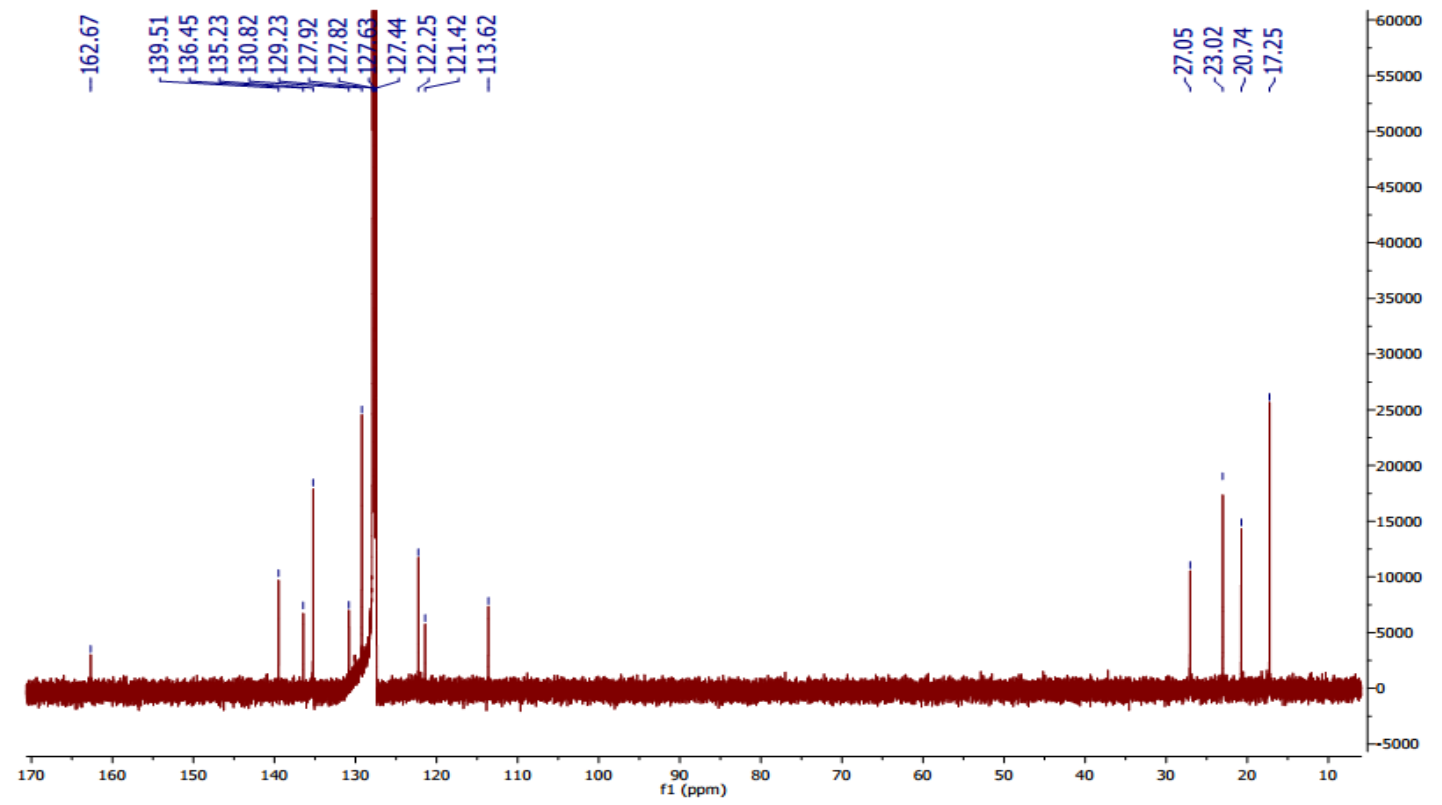

Figure S8. ${ }^{13} \mathrm{C}$ NMR spectra of complex 5.

Table S1. Data in the polymerization of propylene, 1-hexene and 1-octene catalysts 1-4 with different cocatalysts. ${ }^{a}$ 


\begin{tabular}{|c|c|c|c|c|c|c|c|c|c|c|}
\hline entry & catalyst & Ti:Al:TTPB & monomer & $\begin{array}{c}\text { Yield } \\
\text { (gr) }\end{array}$ & $\mathrm{A}^{\mathrm{d}}$ & $\mathrm{M}_{\mathrm{n}}^{\mathrm{e}}$ & $\mathrm{M}_{\mathrm{w}}^{\mathrm{e}}$ & $\bigoplus^{\mathrm{f}}$ & $\begin{array}{c}\mathrm{Ri} \cdot \\
10^{-4 g}\end{array}$ & $\begin{array}{c}\text { Rt. } \\
10^{-7 g} \\
\end{array}$ \\
\hline 1 & 1 & 1:1000:0 & propylene & 2.96 & 3782 & 29,340 & 146,710 & 5.0 & 140 & 201 \\
\hline 2 & $\mathbf{1}$ & 1:500:0 & propylene & 1.17 & 1505 & 70,750 & 312,650 & 4.4 & 56 & 33 \\
\hline 3 & 1 & $1: 11: 1^{c}$ & propylene & 0.64 & 827 & 116,130 & 266,030 & 2.2 & 30 & 11 \\
\hline 4 & 2 & 1:1000:0 & propylene & 3.97 & 4942 & 28,640 & 66,960 & 2.3 & 188 & 277 \\
\hline 5 & 3 & 1:100o:0 & propylene & 0.56 & 702 & 21,600 & 106,050 & 4.9 & 26 & 260 \\
\hline 6 & 4 & 1:1000:0 & propylene & 0.36 & 423 & 58,140 & 168,070 & 2.9 & 17 & 12 \\
\hline 7 & 5 & 1:1000:0 & propylene & 0.01 & 23 & 80,430 & 438,660 & $5 \cdot 4$ & 0.7 & 0.4 \\
\hline 8 & 1 & 1:1000:0 & 1-hexene & 0.54 & 690 & 1,550 & 3,240 & 2.0 & 13 & 697 \\
\hline 9 & $\mathbf{1}$ & 1:500:0 & 1-hexene & 0.21 & 270 & 1,270 & 4,730 & 3.7 & 5 & 334 \\
\hline 10 & 1 & $1: 11: 1^{\mathrm{c}}$ & 1-hexene & 0.57 & 728 & 13,060 & 38,470 & 2.9 & 13 & 87 \\
\hline 11 & 2 & 1:11:1 ${ }^{\mathrm{C}}$ & 1-hexene & 0.48 & 597 & 23,170 & 65,350 & 2.8 & 11 & 41 \\
\hline 12 & 3 & $1: 11: 1^{\mathrm{C}}$ & 1-hexene & 0.46 & 572 & 19,240 & 71,290 & 3.7 & 11 & 48 \\
\hline 13 & 4 & $1: 11: 1^{\mathrm{c}}$ & 1-hexene & 0.04 & 50 & $\begin{array}{l}65,500 \\
1,080^{h}\end{array}$ & $\begin{array}{c}114,200 \\
3770^{h}\end{array}$ & 1.7 & $\begin{array}{l}0.9 \\
0.0\end{array}$ & 1.2 \\
\hline 14 & 5 & $1: 11: 1^{\mathrm{C}}$ & 1-hexene & traces & traces & - & - & - & - & - \\
\hline 15 & 1 & 1:5oo:o & 1-octene & 0.16 & 214 & 1,400 & 5,340 & 3.8 & 3 & 238 \\
\hline 16 & 1 & $1: 11: 1^{\mathrm{c}}$ & 1-octene & 0.57 & 728 & 18,810 & 46,130 & 2.4 & 9 & 60 \\
\hline 17 & 2 & $1: 11: 1^{\mathrm{C}}$ & 1-octene & 0.57 & 709 & 16,850 & 47,6710 & 2.8 & 9 & 67 \\
\hline 18 & 3 & $1: 11: 1^{\mathrm{C}}$ & 1-octene & 0.46 & 572 & 22,020 & 78,050 & $3 \cdot 5$ & 7 & 42 \\
\hline 19 & 4 & $1: 11: 1^{c}$ & 1-octene & 0.21 & 247 & 16,920 & 62,840 & $3 \cdot 7$ & 3 & 25 \\
\hline 20 & 5 & $1: 11: 1^{\mathrm{c}}$ & 1-octene & traces & traces & - & - & - & - & - \\
\hline
\end{tabular}

${ }^{\mathrm{a} C}$ Conditions: for propylene: $15 \mathrm{~mL}$ propylene, $6 \mathrm{~mL}$ toluene, temperature $25^{\circ} \mathrm{C}$, catalyst $5 \mathrm{mg}, 5$ minutes. for 1-hexene and 1-octene: $1 \mathrm{~mL}$ monomer, $0.1 \mathrm{~mL}$ toluene, temperature $25^{\circ} \mathrm{C}$, catalyst $5 \mathrm{mg}, 5$ minutes. ${ }^{\mathrm{b}}$ Molar ratio of Ti:MAO:TTPB. ${ }^{\mathrm{c}}$ Molar ratio of Ti:TIBA:TTPB ${ }^{\mathrm{d} A c t i v i t y}=\mathrm{g}$ Polymer $/ \mathrm{mmol} \mathrm{Ti} \cdot \mathrm{h} .{ }^{\mathrm{e}}$ GPC data in $1,2,4$-trichlorobenzene at $165^{\circ} \mathrm{C}$. Dispersity, ${ }^{\mathrm{g}} \mathrm{mol} / \mathrm{min}$. ${ }^{\mathrm{h}} 8 \%$ of total fractions. 


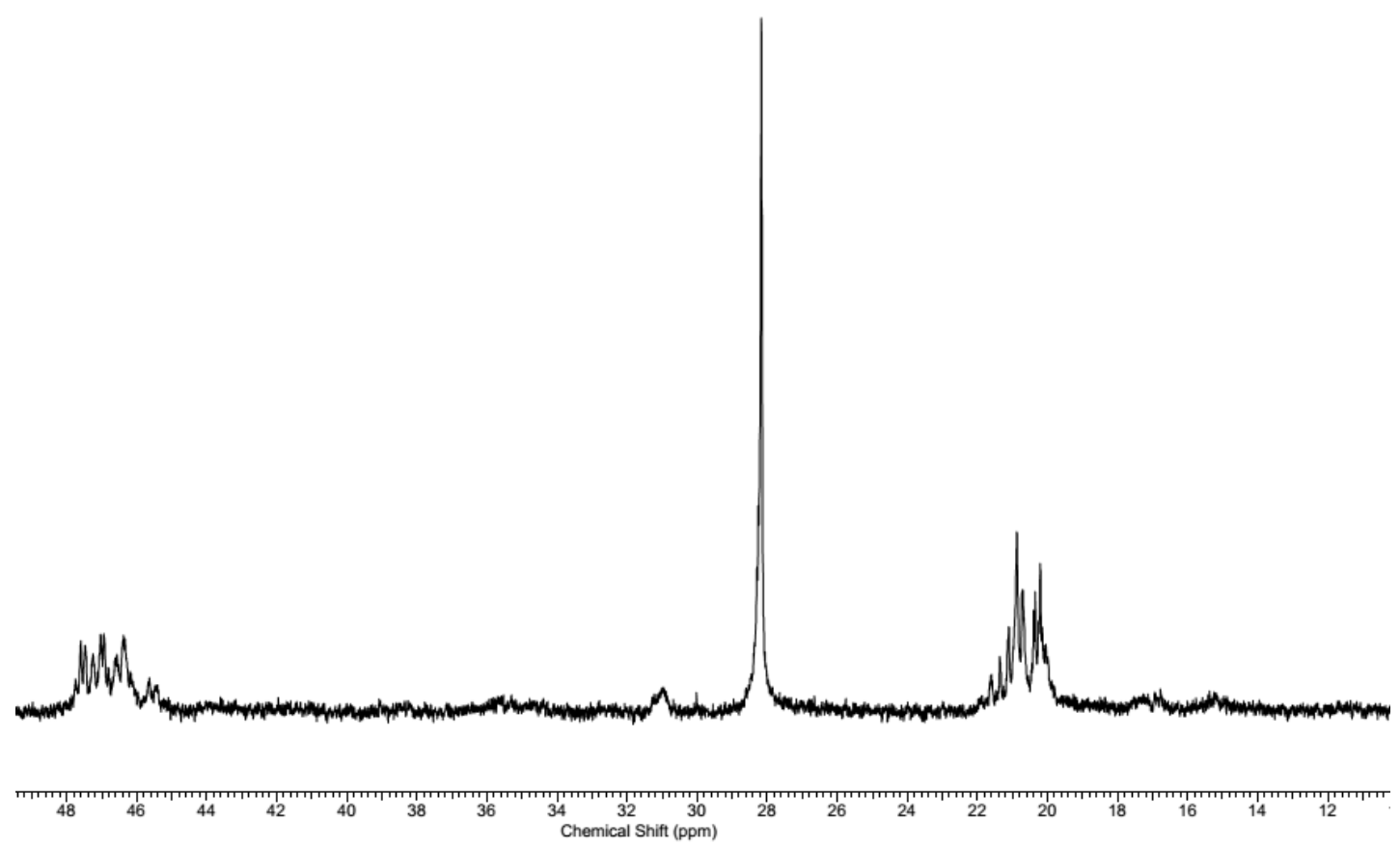

Figure S9. ${ }^{13} \mathrm{C}$ NMR of atactic polypropylene obtained with complex 1, entry 1 (Table S1).

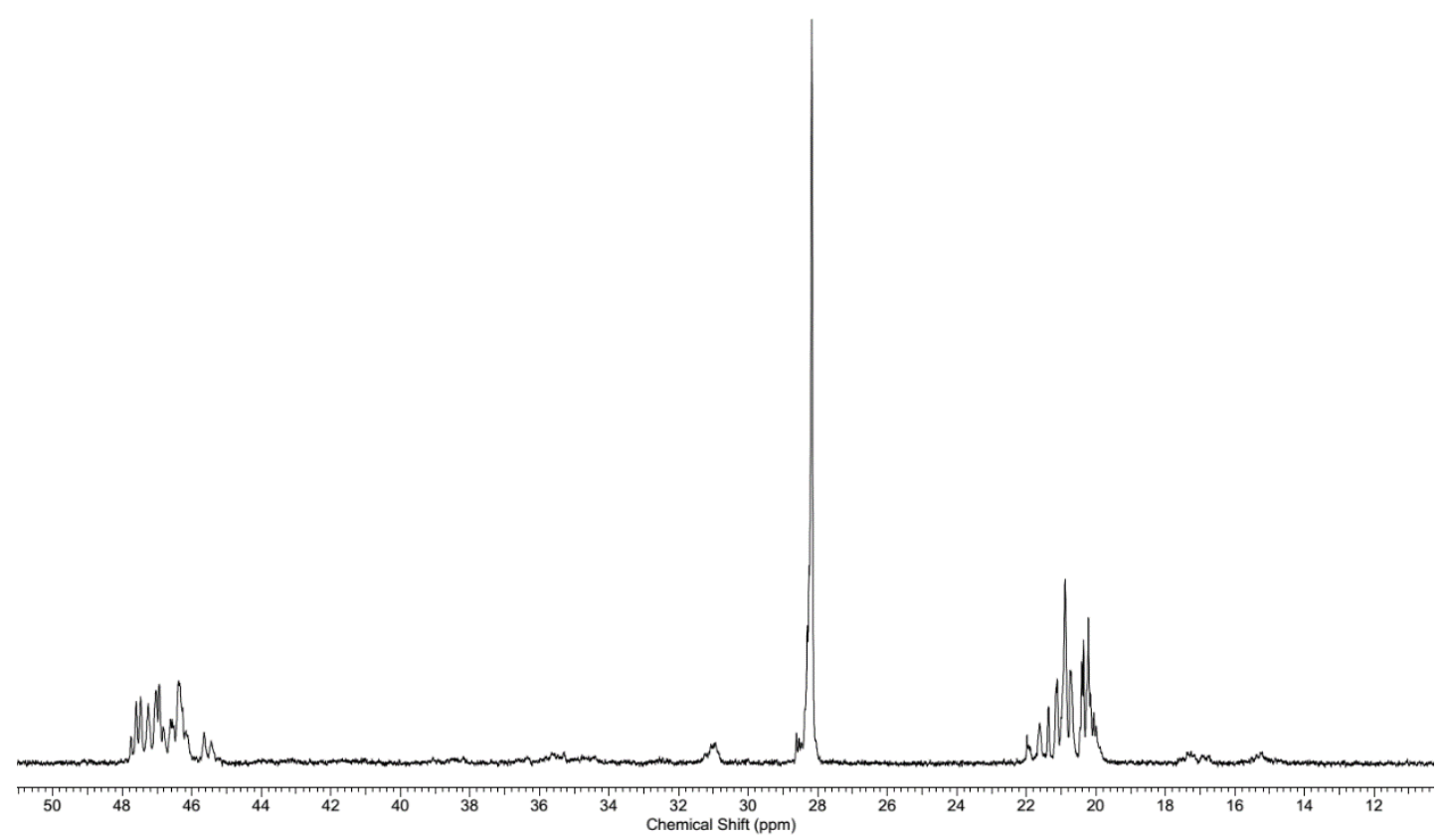

Figure S1o. ${ }^{13} \mathrm{C}$ NMR of atactic polypropylene obtained with complex 1, entry 3 (Table S1). 


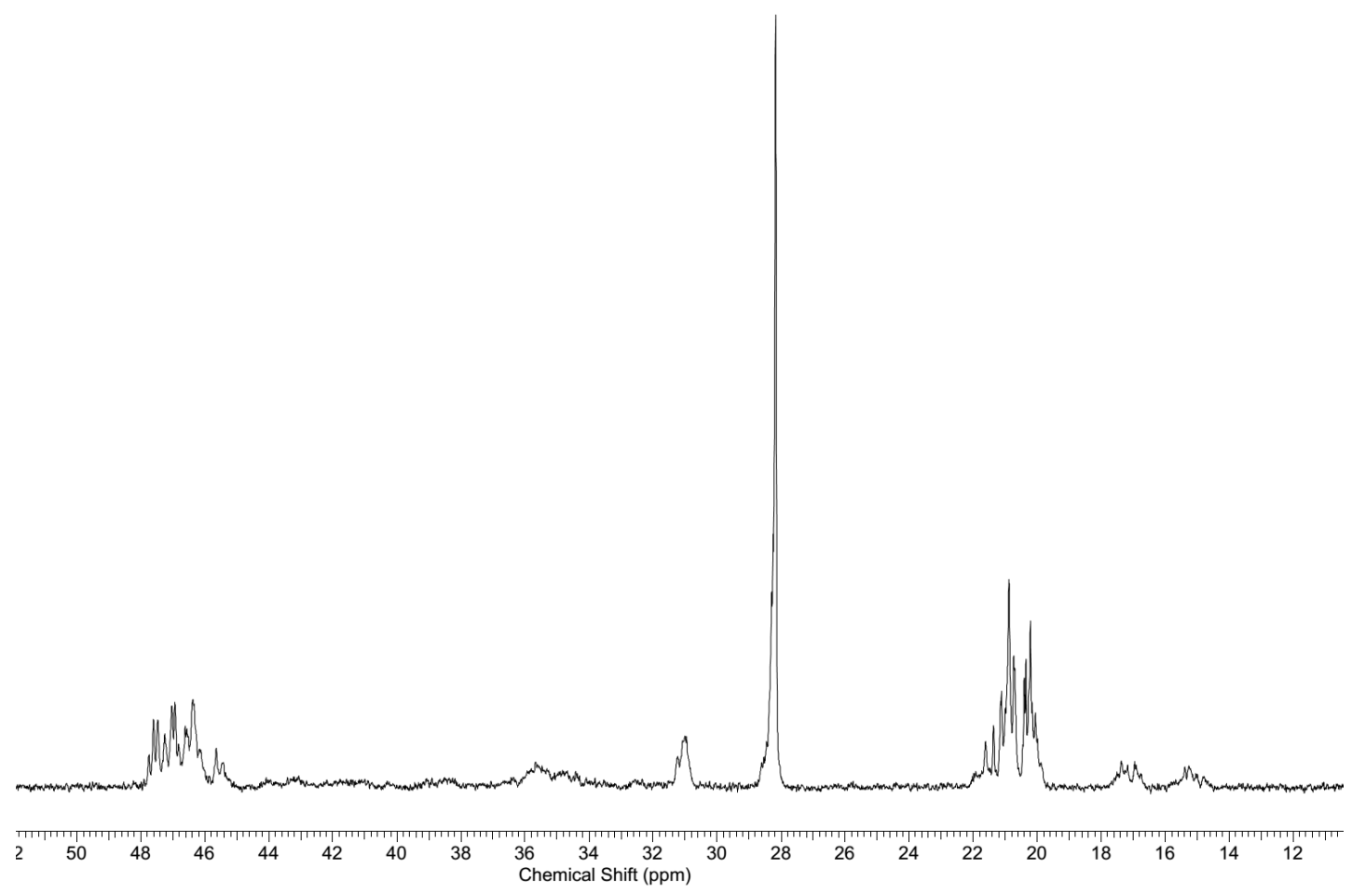

Figure S11. ${ }^{13} \mathrm{C}$ NMR of atactic polypropylene obtained with complex 2, entry 4 (Table S1).

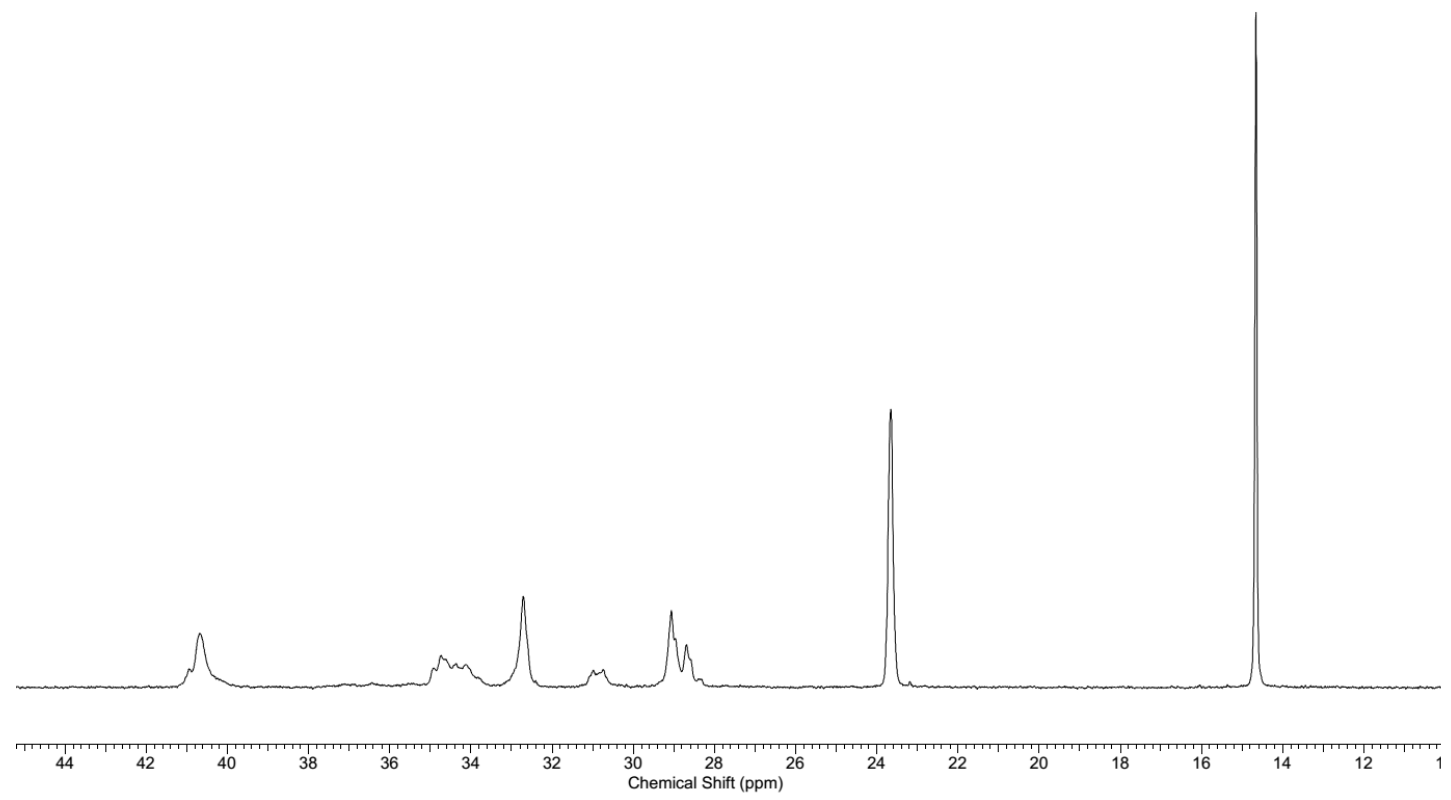

Figure $\mathrm{S}_{12} .{ }^{13} \mathrm{C}$ NMR of atactic poly(1-hexene) obtained with complex 2, entry 11 (Table S1). 


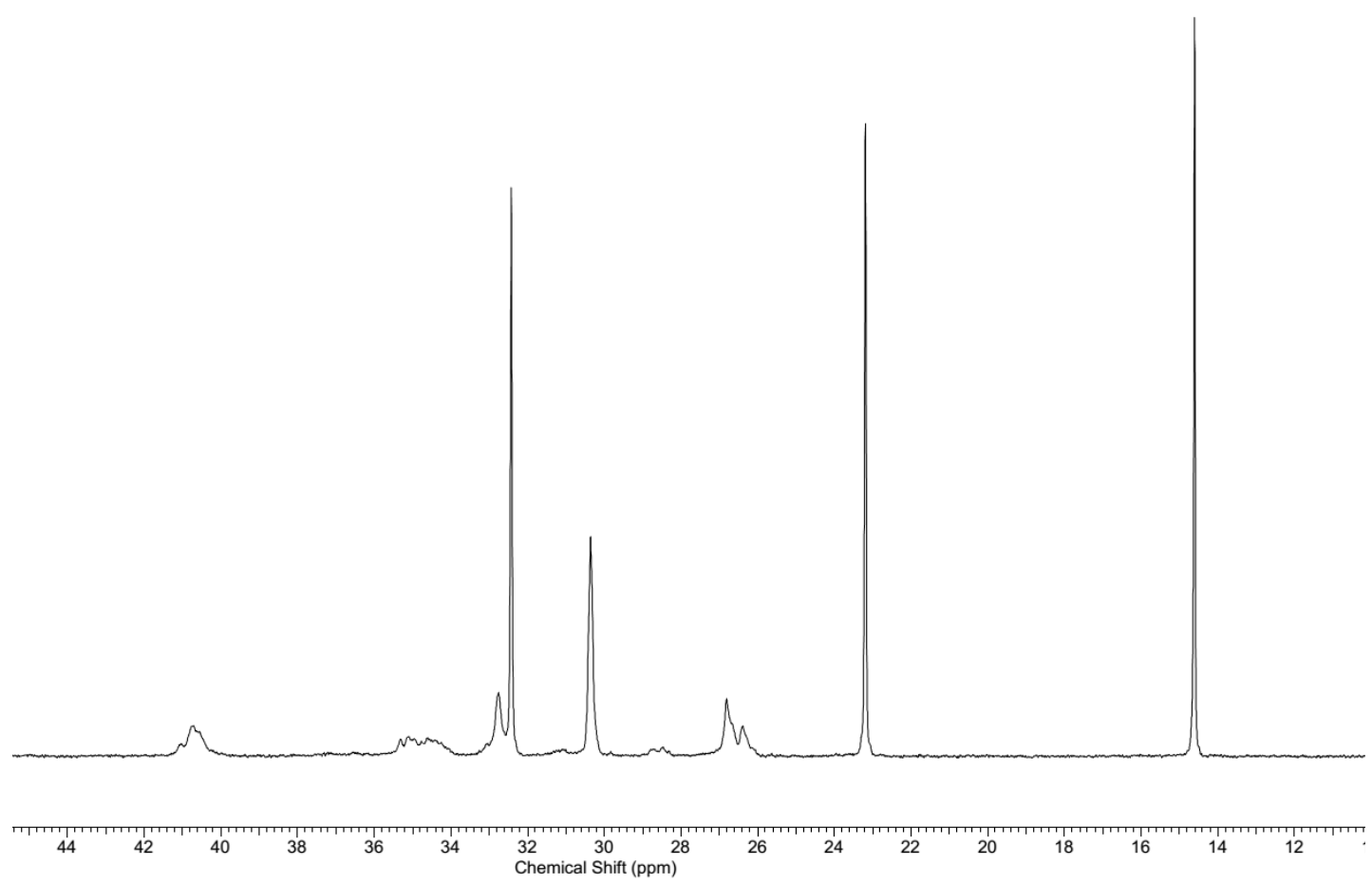

Figure S13. ${ }^{13} \mathrm{C}$ NMR of atactic poly(1-octene) obtained with complex 2, entry 17 (Table S1).

Equations of rate of insertion $\left(\mathrm{R}_{\mathrm{i}}\right)$ and rate of termination $\left(\mathrm{R}_{\mathrm{t}}\right)$ :

$$
\begin{aligned}
R_{i} & =\frac{m / M \cdot W}{t} \\
R_{t} & =\frac{m / M_{n}}{t}
\end{aligned}
$$

$\mathrm{m}$ - Mass of polymer in $\mathrm{g}$

M.W- Molecular weight of monomer in $\mathrm{g} / \mathrm{mol}$.

t- Polymerization time in minutes.

$\mathrm{M}_{\mathrm{n}}$ - Number average molecular weight in $\mathrm{g} / \mathrm{mol}$. 